Article

\title{
Genome Wide Analysis of GH Gene Family Reveals Vvgh9 Positively Regulates Sugar Accumulation under Low Sugar Content in Grape
}

\author{
Guangzhao Xu ${ }^{1,2,+} \mathbb{D}$, Yi Wang ${ }^{1,+}$, Chong Ren ${ }^{1}$, Peige Fan ${ }^{1}$, Yangfu Kuang ${ }^{1,2}$, Yue Wang ${ }^{1,2}$ \\ and Zhenchang Liang ${ }^{1, *}$
}

check for

updates

Citation: Xu, G.; Wang, Y.; Ren, C.; Fan, P.; Kuang, Y.; Wang, Y.; Liang, Z. Genome Wide Analysis of GH Gene Family Reveals Vvgh9 Positively Regulates Sugar Accumulation under Low Sugar Content in Grape. Horticulturae 2021, 7, 453. https://doi.org/10.3390/ horticulturae7110453

Academic Editor: Luigi De Bellis

Received: 20 September 2021

Accepted: 27 October 2021

Published: 3 November 202

Publisher's Note: MDPI stays neutral with regard to jurisdictional claims in published maps and institutional affiliations.

Copyright: (C) 2021 by the authors Licensee MDPI, Basel, Switzerland. This article is an open access article distributed under the terms and conditions of the Creative Commons Attribution (CC BY) license (https:// creativecommons.org/licenses/by/ $4.0 /)$.
1 Beijing Key Laboratory of Grape Science and Enology, CAS Key Laboratory of Plant Resources, Institute of Botany, The Chinese Academy of Sciences, Beijing 100093, China; guangzhao90@163.com (G.X.); wangyi19881107@163.com (Y.W.); chongr@ibcas.ac.cn (C.R.); fanpg@ibcas.ac.cn (P.F.); kuangyf@ibcas.ac.cn (Y.K.); qiuyingwy@163.com (Y.W.)

2 University of Chinese Academy of Sciences, Beijing 100049, China

* Correspondence: zl249@ibcas.ac.cn; Tel.: +86-010-62836064

+ These authors contributed equally to this work.

\begin{abstract}
Sugar content directly affects grape (Vitis vinifera L.) berry quality and the resulting wine Therefore, it is of great importance to study and explore novel genes that affect sugar accumulation in grapes. Glycosyl hydrolases (GHs) are key enzymes hydrolyzing polysaccharides into monosaccharides and play important roles in the regulation of carbohydrate metabolism. Nevertheless, the impact of GHs on the regulation of sugar accumulation in plants has rarely been investigated. In this study, we identified 11 putative GH genes in grapevines by phylogeny analysis. RNA-seq and quantitative real-time PCR results demonstrated that the expression level of $V v G H 9$ was higher during the fruit set stage, which had lower sugar content than the véraison and ripe stages. Treatment of grape berries with exogenous sugar two weeks before véraison revealed that $V v G H 9$ was rapidly induced by sucrose, fructose, and glucose. When ' $41 \mathrm{~B}^{\prime}$ calli was treated with different concentrations of glucose, $V v G H 9$ expression increased at first and then decreased with the increase of glucose concentration. Overexpression of $V v G H 9$ in grape calli and tomatoes also confirmed that this gene could contribute to sugar accumulation. All the above results demonstrated that $V v G H 9$ promotes sugar accumulation under low sugar content in plants.
\end{abstract}

Keywords: grape; sugar; $V v G H 9$; transcriptomic analysis; overexpression

\section{Introduction}

As one of the fruit crops with the most extensively cultivated area and the longest industrial chain in the world [1], grapes (Vitis vinifera) substantially contribute to the global economy. Besides the water content, sugars are also the primary substances present in mature grape berries, accounting for $15-25 \%$ of the fresh berries [2]. The type and content of sugars not only affect the sensory quality of grape berries, but also affect the abundance and flavor of the wine. The predominant sugars in grape berries are glucose and fructose, while sucrose is present but in low concentrations. Studies have shown that sugar not only acts as a carbon and energy source, but also as a regulatory signal of genes expression, plant growth, and development [3]. Therefore, it is important to analyze the molecular mechanisms underlying the direct and indirect regulation in sugar accumulation of plants.

GHs (glycosyl hydrolases) are key enzymes that hydrolyze glycosidic bonds of carbohydrates and a noncarbohydrate moiety in all living organisms [4]. McCarter et al. [5] investigated the catalytic mechanism of polysaccharide hydrolysis through structural studies of retaining $\beta$-glycosyl hydrolases. Heritable deficiencies of glycosyl hydrolases in human may result in lactose intolerance [6] and lysosomal storage diseases [7]. Bauer 
et al. [8] summarized the developments and potential functions of GH proteins in hyperthermophilic microorganisms. In 1999, it was first reported that glycosyl hydrolases, which recognize different glycofuranoside residues, share a common sequence motif [9]. It was reported that the activity of O-glycosyl hydrolases was increased by the presence of $1 \rightarrow 3-\beta$-D-glucanase inhibitors [10]. Moreover, various GH enzymes are also widely applied in the food industry [11]. It was also assumed that GH may be effectively employed as synthetic enzymes in the near future based on the enzyme catalytic scheme and the development of nonaqueous enzymology [12]. Meanwhile, some GH family 1 members were reported to be involved in response to hormone treatment and abiotic stresses $[13,14]$.

Additionally, GHs are widely used as a natural hydrolytic agent to make raw complex mixtures into value-added simple materials [15]. Since the end of the last century, engineered GHs have become a very helpful biomaterial to improve the efficiency and yields of glycosylation reactions [16]. In 2015, Trincone summed up two possible experimental protocols, the reverse hydrolysis procedure and the kinetic approach, for the use of GHs in synthesis [17]. In many fruits, such as apples [18] and tomatoes [19], starch mainly accumulates at early fruit developmental stages and is degraded into simple sugars during fruit development. It was reported that a gene encoding an $\alpha$-glucosidase, AdAGL3, was significantly induced by ethylene and promoted starch degradation [20]. As GH family members, $\beta$-glucosidases can hydrolyze the $\beta$-D-glycosidic bonds of glucosides and oligosaccharides non-reducing end to release glucose [21]. A recent study reported that MaGlu1A, a new gene encoding $\beta$-glucosidase MaGlu1A, strongly responded to the stimulation of supplemental glucose and the active center of MaGlu1A was identified by site-directed mutagenesis [22]. To date, the function of GH genes in sugar accumulation, particularly in grapevines, is still rarely studied.

In this study, we carried out a genome-wide analysis of $G H$ genes in $V$. vinifera and measured $G H$ genes expression levels at three berry developmental stages belonging to 22 varieties. We also explored their function and regulation mechanism in sugar accumulation. This study is an insight into the function of the GH family and provides new molecular markers for the high-sugar-content breeding of grapes.

\section{Materials and Methods}

\subsection{Plants and Growth Conditions}

Five different stages (FS: fruit set; T: touching; V: véraison; M: mid-ripening; R: ripe) of $V$. vinifera cv. 'Muscat Hamburg' berries were collected to measure sugar and VvGH9 gene expression levels. For this purpose, berries of V. vinifera $\mathrm{cv}$. 'Chardonnay' were used for exogenous sugar treatment two weeks before véraison. Seedlings of 'Muscat Hamburg' and 'Chardonnay' were planted in the vineyard at the Institute of Botany, Chinese Academy of Sciences, Beijing, all these plants were well planted and were in good condition. '41B' $(V$. vinifera $\times V$. berlandier) calli were cultured according to the protocol described by Wang et al. [23].

\subsection{Identification and Phylogenetic Analysis of GH Genes}

Hidden Markov Model (HMM) (PF01055.26) was used to identify GH proteins. The obtained proteins were also searched against the whole proteins (IGGP V2.1) of the grape by BLAST. Then the candidate members from HMM and BLAST were confirmed by NCBIConserved Domain Data (CDD) search (http:/ / www.ncbi.nlm.nih.gov/Structure/cdd/ wrpsb.cgi, accessed on 21 November 2020).

Protein sequences of GH family members were used to construct the phylogenetic tree. These sequences were aligned by MUSCLE first, then the construction of the phylogenetic tree was performed by MEGA 7.0 software (https: / / www.megasoftware.net/, accessed on 17 July 2021) [23] with the method of neighbor-joining and bootstrap of 1000 [24]. 


\subsection{Gene Location and Duplication Analysis of GH Genes in the Grape Genome}

The locations and duplication events analysis of $G H$ genes was performed according to $\mathrm{Xu}$ et al. [25]. The location of each gene was shown on CIRCOS map [26], and the red curves and the color dots represented segment and tandem duplication events respectively.

\subsection{RNA-seq Data Analysis}

The expression pattern of $V v G H 9$ at 3 developmental stages in 22 grape varieties (details are listed in Tables S1 and S2) was obtained from the grape-RNA database (http:/ /www.grapeworld.cn/gt/, accessed on 9 May 2021) [27].

\subsection{Total RNA Extraction and Quantitative Real-Time PCR ( $q R T-P C R)$ Assays}

The total RNA of 'Muscat Hamburg' berries, 'Chardonnay' berries and '41B' calli was extracted by HiPure HP Plant RNA Mini Kit (Magen, Guangzhou, China). HiScript III 1st Strand cDNA Synthesis Kit (R312-02, Vazyme, Nanjing, China) was used to synthesize the first cDNA strand, and cDNA for qRT-PCR was generated by HiScript II Reverse Transcriptase (R201-02, Vazyme, Nanjing, China). The qRT-PCR primers of VvGH9 and a reference gene VvACTIN (Accession: EC969944) are listed in Table S3. The specific methods for qRT-PCR were performed according to Wang et al. [23].

\subsection{Exogenous Sugar Treatment of Grape Berry via Injection}

About 2 weeks before véraison $(\mathrm{V})$, three bunches of 'Chardonnay' berries with similar numbers and types for each soluble sugar were selected. Sucrose, glucose, and fructose solutions (each $20 \mathrm{~g} / \mathrm{L}$ ) were separately placed into injection bags. A needle with $1 \mathrm{~mm}$ diameter was inserted into about $1 \mathrm{~cm}$ depth, and the flow rate was about $0.1 \mathrm{~mL} / \mathrm{min}$. Subsequently, the expression levels of VvGH9 were analyzed at $0,12,24$, and $48 \mathrm{~h}$.

\subsection{Treatment of Grape Calli with Different Concentrations of Glucose}

The ' $41 \mathrm{~B}$ ' calli were used for sugar treatment, and the culture method was performed as described by Wang et al. [23]. For different glucose concentrations treatments, the calli were cultured in a corresponding liquid medium with different concentrations of glucose for 7 days. Next, the expression patterns of $V v G H 9$ in these materials were analyzed.

\subsection{Cloning of VvGH9 Gene}

The coding sequence of $V v G H 9$ (VIT_202s0033g01410, also known as alpha-glucosidase 2-like in NCBI) was amplified using the 'Chardonnay' leaves cDNA as template with the primers VvGH9-F/VvGH9-R (Table S3), designed according to the predicted coding sequence of $V v G H 9$ from $V$. vinifera cv. 'Pinot Noir' (PN40024) [28]. The PCR was conducted with KOD-Plus-Neo (Toyobo, Osaka, Japan) DNA polymerase in a total volume of $50 \mu \mathrm{L}$ at $95{ }^{\circ} \mathrm{C}$ for $2 \mathrm{~min}$; 40 cycles of $98{ }^{\circ} \mathrm{C}$ for $10 \mathrm{~s}, 56{ }^{\circ} \mathrm{C}$ for $30 \mathrm{~s}, 68{ }^{\circ} \mathrm{C}$ for $90 \mathrm{~s} ; 68{ }^{\circ} \mathrm{C}$ for $10 \mathrm{~min}$. The product of PCR was cloned into pLB-Simple vector (Tiangen, Beijing, China) and analyzed by Sanger sequencing. The verified sequence was then used as the template to amplify the open-reading frame (ORF) of $V v G H 9$ using the primers GH9-ORF-F/GH9ORF-R (Table S3), and the PCR was performed as described above. The amplified VvGH9 ORF fragment was ligated into the KpnI (NEB, Ipswich, MA, USA)-digested pCAMBIA2300 vector, which serves as an overexpression vector modified by Wang et al. [23], through the homologous recombination methods using SE Seamless Cloning and Assembly Kit (ZOMANBIO, Beijing, China).

\subsection{Transformation of Grapevine Calli and Tomato}

The recombinant vector above and the empty vector (EV) were then transformed into ' $41 \mathrm{~B}^{\prime}$ ' calli as described by $\mathrm{Xu}$ et al. [25]. Then, $V v G H 9$-transgenic tomatoes were generated to further investigate its functions, and the transformation method was based on Sun et al. [29]. Using gene/kanamycin-specific primers (Table S3) for PCR to confirm the transgenic plants. 


\subsection{Sugar Determination}

The frozen grape berries or calli $(0.2 \mathrm{~g}$ fresh weight $(\mathrm{FW}))$ were homogenized into $2 \mathrm{~mL}$ deionized water for $4 \mathrm{~h}$ in an ice bath with intermittent mixing, and then were centrifuged $\left(8000 \times g, 10 \mathrm{~min}, 4^{\circ} \mathrm{C}\right)$. Specific filtration and analytical methods were performed according to Zhang et al. [30].

\section{Results}

\subsection{Identification and Phylogenetic Analysis of Eleven GH Genes in Grape}

In total, $11 \mathrm{GH}$ genes named $V v G H 1$ to $V v G H 11$ were identified in the grapevine genome (12X, V2.1) (Figure 1). According to the phylogenetic tree of $11 \mathrm{VvGH}$ proteins (Figure 1), VvGH members were classified into four groups (GH_1, GH_2, GH_3, and GH_4). The GH_1 was the largest group, containing 4 members, while the GH_4 was the smallest group, with only one member.

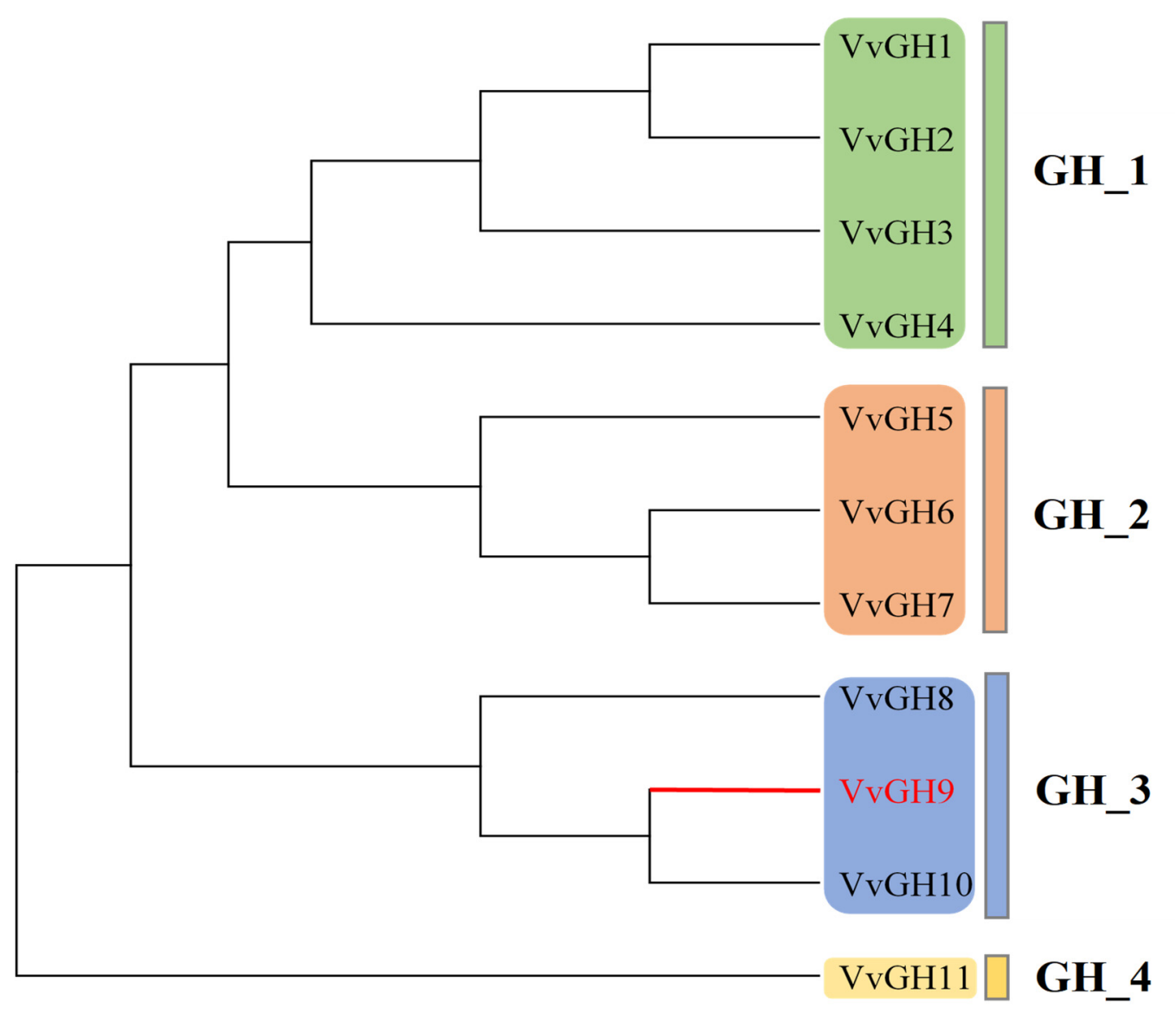

Figure 1. Phylogenetic analysis of GH genes in $V$. vinifera. The unrooted tree was generated by ClustalW in MEGA7 using the conserved amino acid sequences of the $11 \mathrm{~V}$. vinifera $\mathrm{GH}$ proteins.

\subsection{Chromosome Localization and Gene Duplication Analysis of Vvgh Genes}

The $11 \mathrm{VvGH}$ genes were distributed on seven chromosomes including chromosome 1 , 2, 5, 8, 10,13, and 16 (Figure 2). Chromosome 2 contained four $V v G H$ genes, chromosome 10 contained two $V v G H$ genes, and each of the other chromosomes contained the $1 V v G H$ gene respectively. Three segmental duplication events of $G H$ genes were identified in $V$. vinifera, and eight members of the $V v G H$ genes were related to these events. Moreover, four genes ( $V v G H 4, V v G H 10$ and $V v G H 6, V v G H 7$ ) were involved in two tandem duplication events. These four members were involved in both segmental and tandem duplication and three members of $\mathrm{VvGH}$ genes were not detected in the duplication events. 


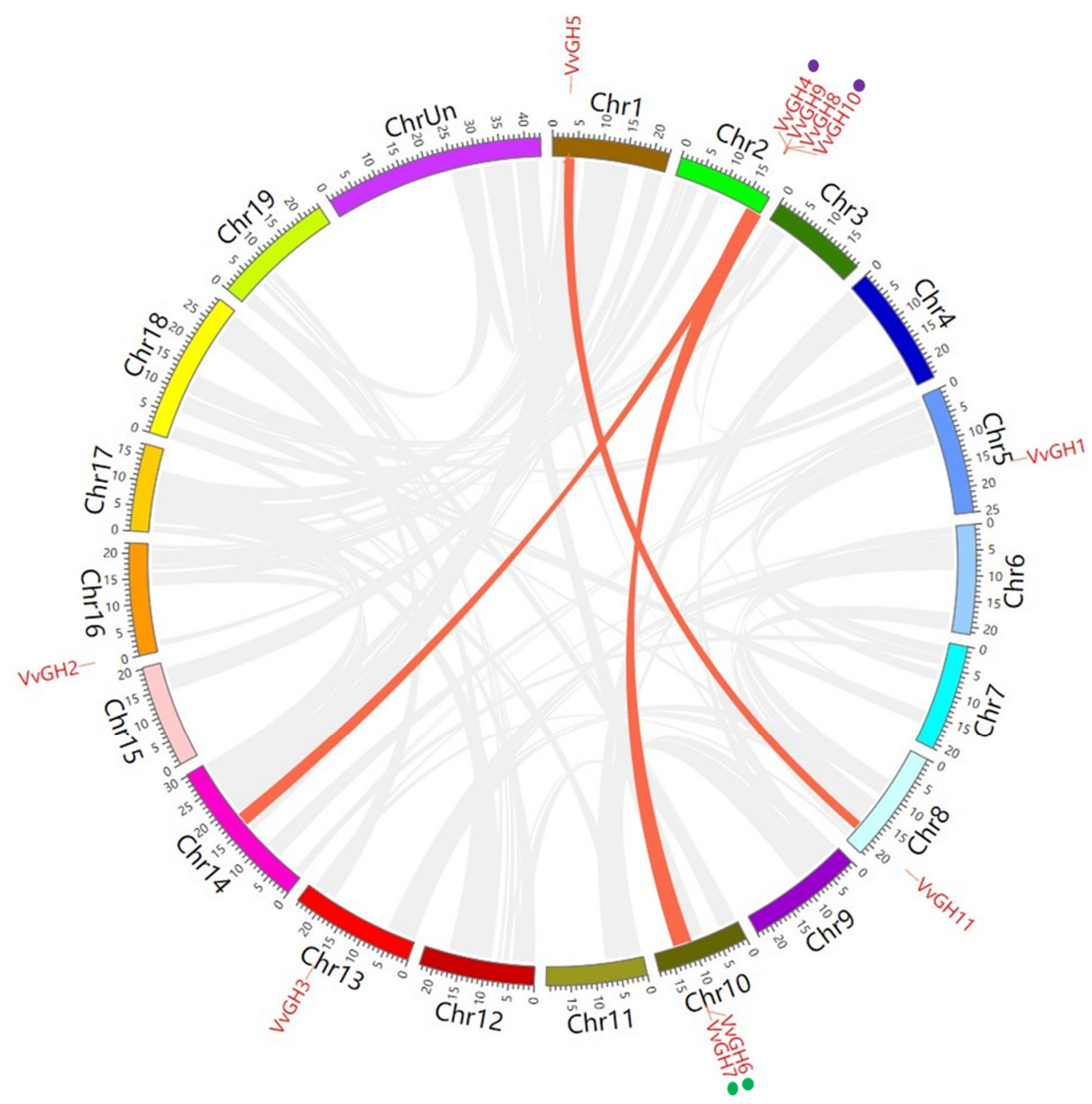

Figure 2. Chromosome distribution and duplication analysis of grapevine GH genes. Chromosomes 1-19 are shown in different colors and a circular form. The approximate distribution of each $V v G H$ gene is marked with a short red line on the circle. Red curves denote the details of segmental duplication between grape GH genes. Different colorful dots denote the details of tandem duplication between grapevine $G H$ genes.

\subsection{Expression Profiles of Vvgh Genes at Different Developmental Stages in Grape Berries}

To obtain insights into the potential roles of the 11 genes in different developmental stages of grape berry, we estimated their expression patterns based on RNA-seq data (http:/ / www.grapeworld.cn/gt/, accessed on 9 May 2021) of 22 grape varieties (Figure 3). Most $V v G H$ genes had significant expression differences between different stages. $V v G H 5$, $V v G H 6$, and $V v G H 9$ had similar expression patterns in all samples and expressed as significantly different between the first two stages in some varieties. However, only $V v G H 9$ was observed to be expressed differently in the three stages of almost all the 22 grape varieties.

Moreover, six representative varieties were selected to further analyze the three significant differential expressed genes ( $V v G H 5, V v G H 6$, and $V v G H 9$ ), and the results showed that only $V v G H 9$ was expressed and existed a significant differential expression at all stages of the six selected varieties (Figure 4). 


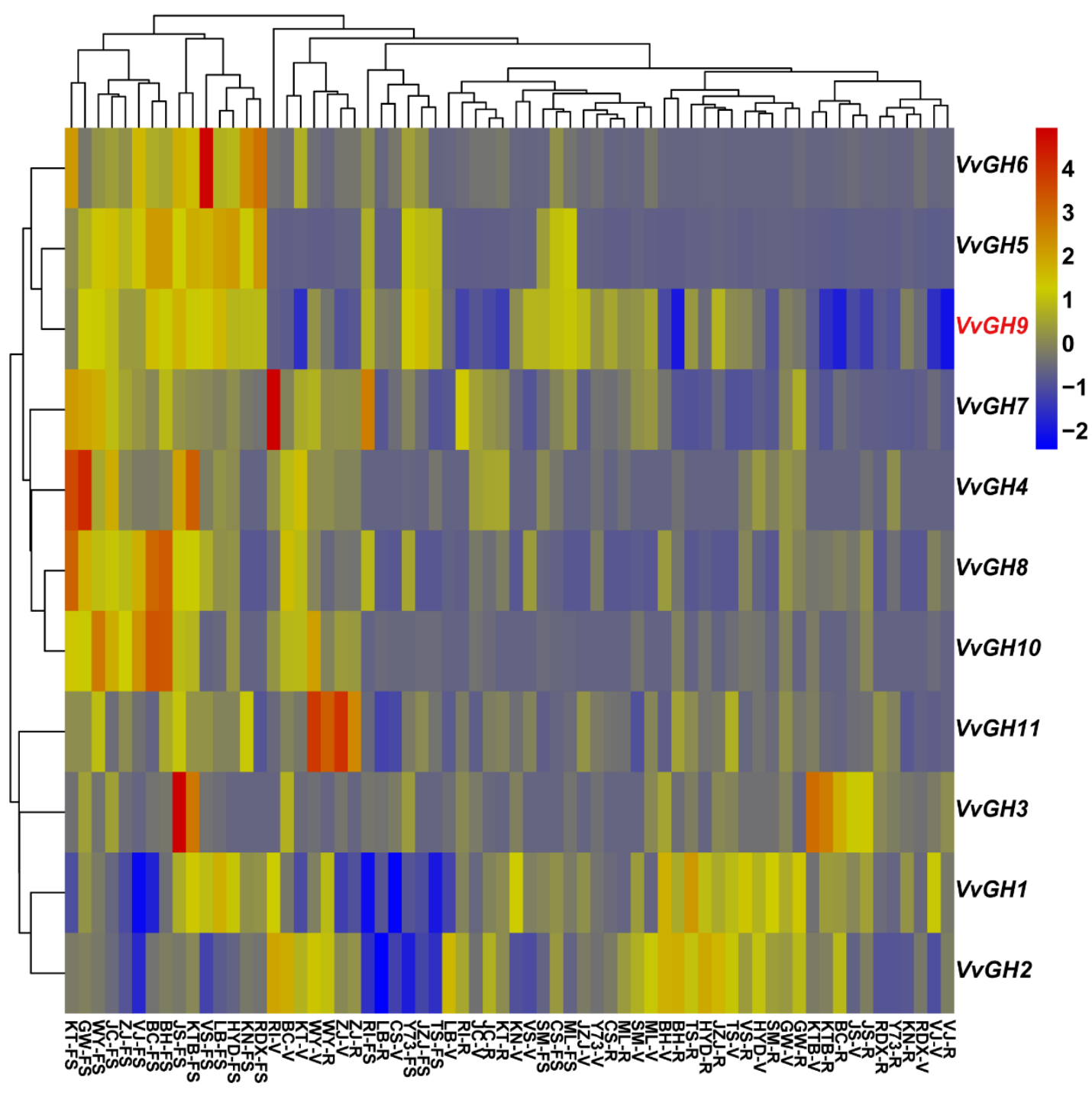

Figure 3. Expression profiles of $V v G H$ genes at 3 developmental stages of 22 grape varieties. The expression levels of these genes were downloaded from the grape-RNA database. The color intensity represents relative expression levels, with red as increased transcript abundance and blue as decreased transcript abundance. The acronyms below the heatmap represent grape varieties (Table S2). FS: fruit set. V: véraison. R: ripe.

To further elucidate the expression patterns of $V v G H 9$ at five developmental stages in the fruits of $V$. vinifera $\mathrm{cv}$. 'Muscat Hamburg' (Figure 5a), we performed an assay of qRTPCR (Figure 5b). According to the qRT-PCR results, VvGH9 exhibited a higher expression level at the fruit set (FS, E-L stage 27) and touching (T, E-L stage 32) [31]. The results of the qRT-PCR were basically consistent with the transcriptomic analysis. We also measured the sugar content of five developmental stages of 'Muscat Hamburg'. The measurement results showed that the sucrose content was too low to be detected in all the developmental periods in 'Muscat Hamburg' berry, while the two monosaccharides (glucose and fructose) content were very low $(<10 \mathrm{mg} / \mathrm{g})$ at FS and T stages but increased rapidly since véraison ( $>30 \mathrm{mg} / \mathrm{g}$ ) (Figure 5c). The general trend of $V v G H 9$ expression pattern was basically opposite to that of total sugar content. 

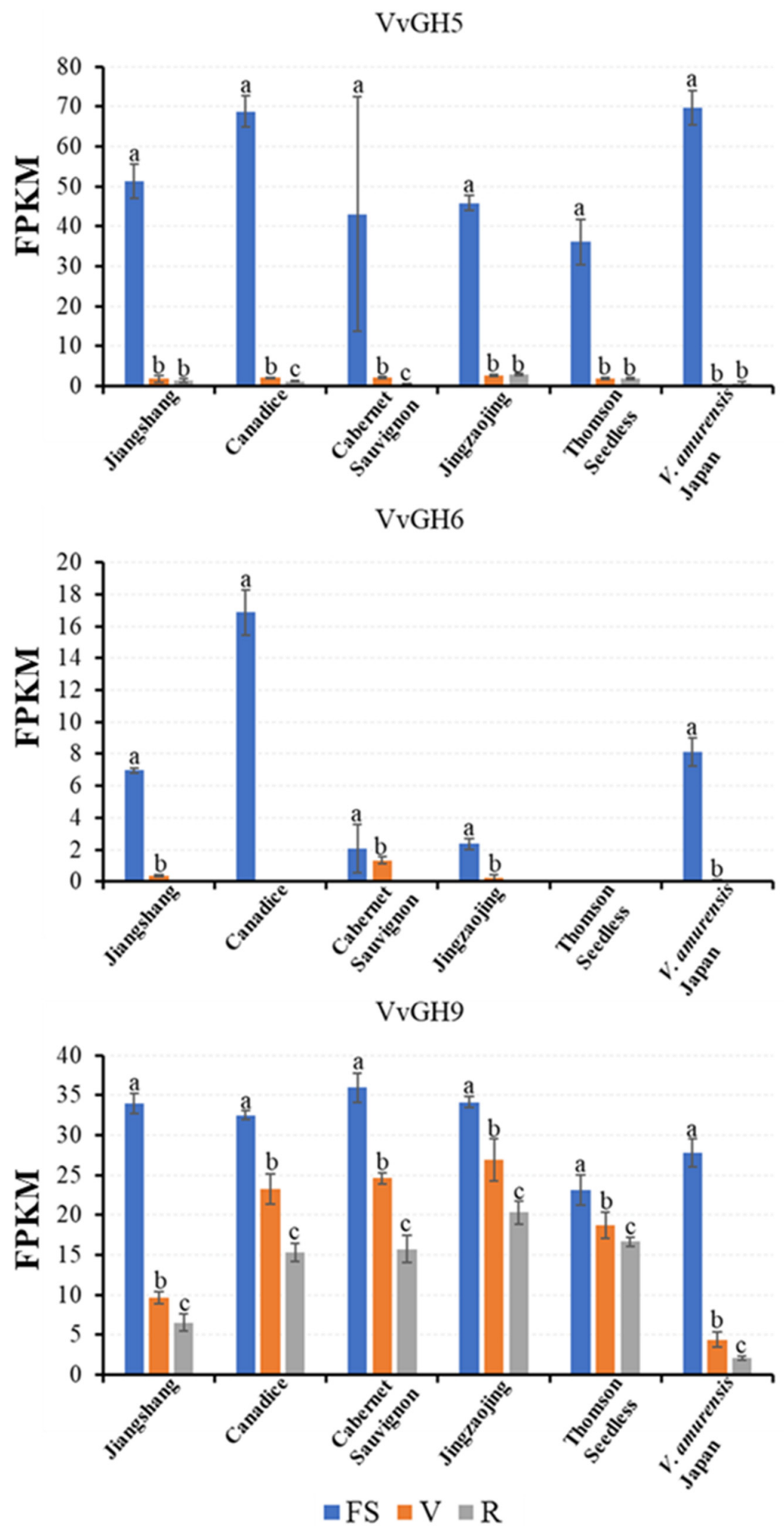

Figure 4. Transcriptomics analysis of $V v G H 5, V v G H 6$, and $V v G H 9$ at three developmental stages of six representative grape varieties berry. The expression levels of the three genes were downloaded from the grape-RNA database. FS: fruit set; V: véraison; R: ripe. The letters above the bars indicated the significant differences by student's $t$-test $(p<0.05)$. Three biological replicates were analyzed, and the error bars represented the SD. 
a
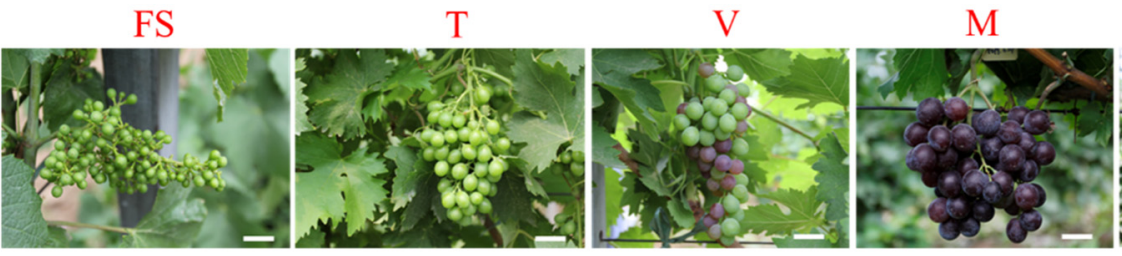

$\mathrm{R}$

b
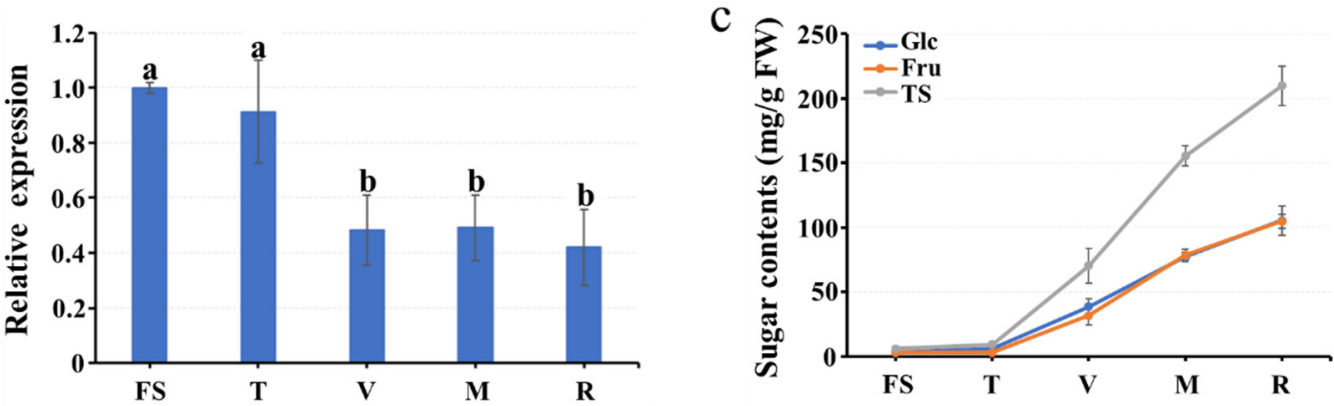

Figure 5. The relative expression level of the $V v G H 9$ gene and the sugar content of 'Muscat Hamburg' grape fruit at five different developmental stages. (a) Fruit growth status in different developmental stages. Scale bars, $2 \mathrm{~cm}$. (b) Relative $V v G H$ expression in different berry developmental stages. (c) Sugar content determination results of grape berries. FS: fruit set; T: touching; V: véraison; M: mid-ripening; R: ripe. FW: fresh weight. The letters above the bars indicated the significant differences by student's $t$-test $(p<0.05)$. Three biological replicates were analyzed, and the error bars represented the SD.

\subsection{Exogenous Sugar Treatment by Injection Improves the Expression Level of Vvgh9 in Grape Berries}

In order to investigate whether $V v G H 9$ responds to different sugars in fruit, $20 \mathrm{~g} / \mathrm{L}$ of sucrose, glucose, and fructose were separately injected into spike-stalk of 'Chardonnay' berry about two weeks before véraison (Figure 6a). Expression of VvGH9 was rapidly induced by treatment with each of the three soluble sugars, with the response to sucrose at $12 \mathrm{~h}$ being strongest (Figure $6 \mathrm{~b}-\mathrm{d}$ ).

a
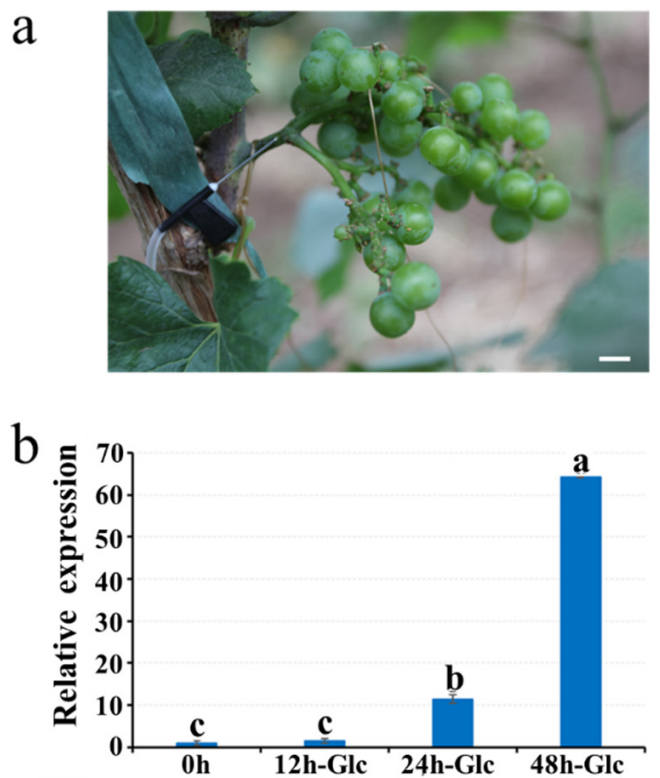
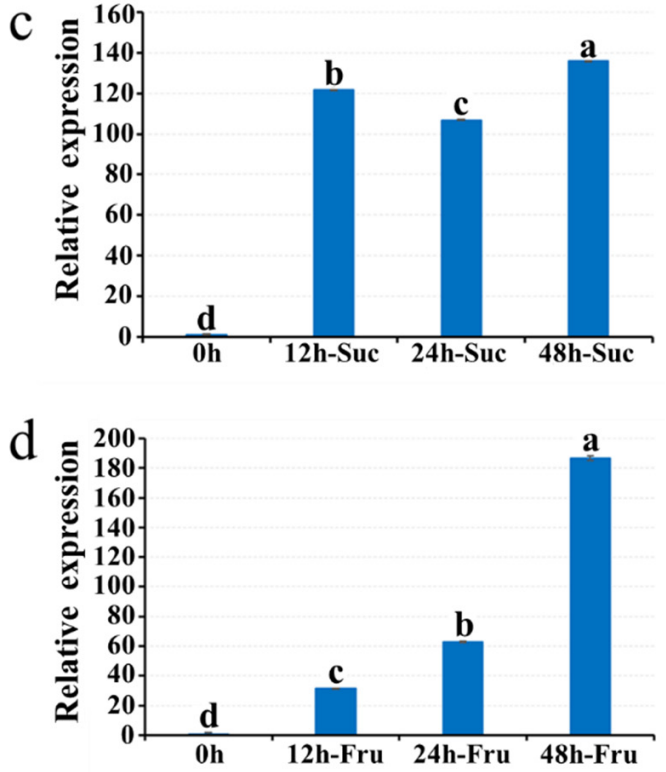

Figure 6. The relative expression profiles of $V v G H 9$ in grape berry injected with sucrose (a), glucose (b), and fructose (c) and treatment of 'Chardonnay' grape berry before véraison with exogenous sugars via injection $(\mathbf{d})$. Scale bars, $1 \mathrm{~cm}$. The letters above the bars indicated the significant differences by student's $t$-test $(p<0.05)$. Three biological replicates were analyzed, and the error bars represented the SD. 
3.5. The Influence of Different Glucose Concentrations on the Expression Pattern of Vvgh9 in '41B' Calli

To explore whether different sugar contents affect the expression pattern of VvGH9 in grapes, we treated ' $41 \mathrm{~B}$ ' calli with different glucose concentrations and detected the expression level of $V v G H 9$. The results revealed that the $V v G H 9$ expression level increased remarkably first and then decreased with the increase of glucose concentration. The expression of $V v G H 9$ reached the highest at $30 \mathrm{mg} / \mathrm{g}$ glucose concentration (Figure 7).

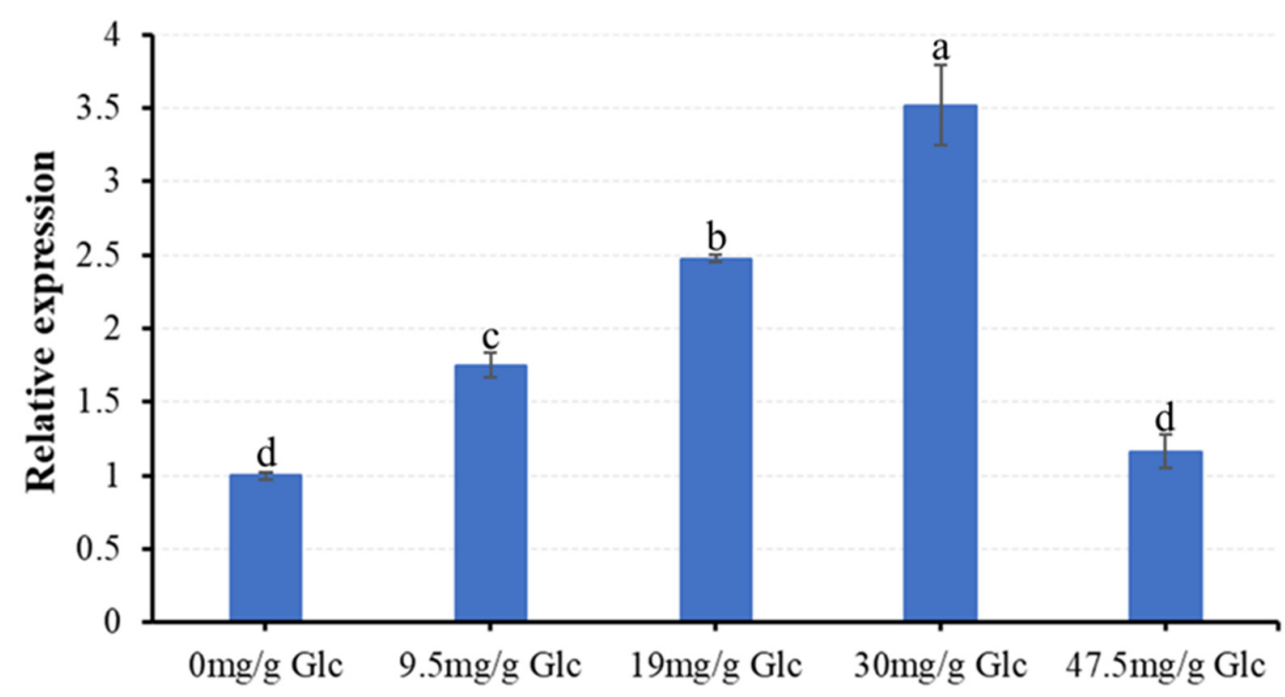

Figure 7. The expression level of $V v G H 9$ in '41B' wild-type calli treated with different concentrations of glucose. The letters above the bars indicated the significant differences by student's $t$-test $(p<0.05)$. Three biological replicates were analyzed, and the error bars represented the SD.

\subsection{Overexpression of VvGH9 Gene Improved Sugar Content in $41 B$ Calli}

In order to investigate whether $V v G H 9$ expression affects the sugar content of grape cells, a 35S::VvGH9 construct was then stably transformed into '41B' calli via Agrobacteriamediated transformation. The successful transformation was confirmed by PCR (Figure 8a). $V v G H 9$ expression in the '41B' calli of overexpressed $V v G H 9$ gene (GH9-OE) was 39 times higher than that of transformed with 35S::pCAMBIA2300 empty vector (EV) (Figure 8b). Sugar analysis of GH-OE and EV by high performance liquid chromatography (HPLC) showed that the contents of sucrose and fructose in GH9-OE were significantly higher than that of EV, while there was no observable difference in glucose content of GH-OE and EV (Figure 8c). The contents of all three sugars were less than $10 \mathrm{mg} / \mathrm{g}$ both in GH-OE and EV.

\subsection{Heterologous Overexpression of Vvgh9 Increased the Sugar Content of Tomato Berries}

To further investigate the function of $V v G H 9$ in fruits, we obtained overexpressed VvGH9 tomato (Lycopersicon esculentum) plants (VvGH9-OE-Le, Figure 9a). The successful transformation was confirmed by PCR (Figure $9 \mathrm{~b}$ ). In addition, we also detected the sugar content of the ripe fruit of VvGH9-OE-Le and wild-type tomatoes (WT-Le). The results showed that $\mathrm{VvGH9}$ markedly increased the content of sucrose, glucose, and fructose in tomato fruits (Figure 9c). Meanwhile, overexpression of $V v G H 9$ could promote the production of sucrose, while the sucrose content was too low to be detected in the fruit of wild-type tomatoes. 

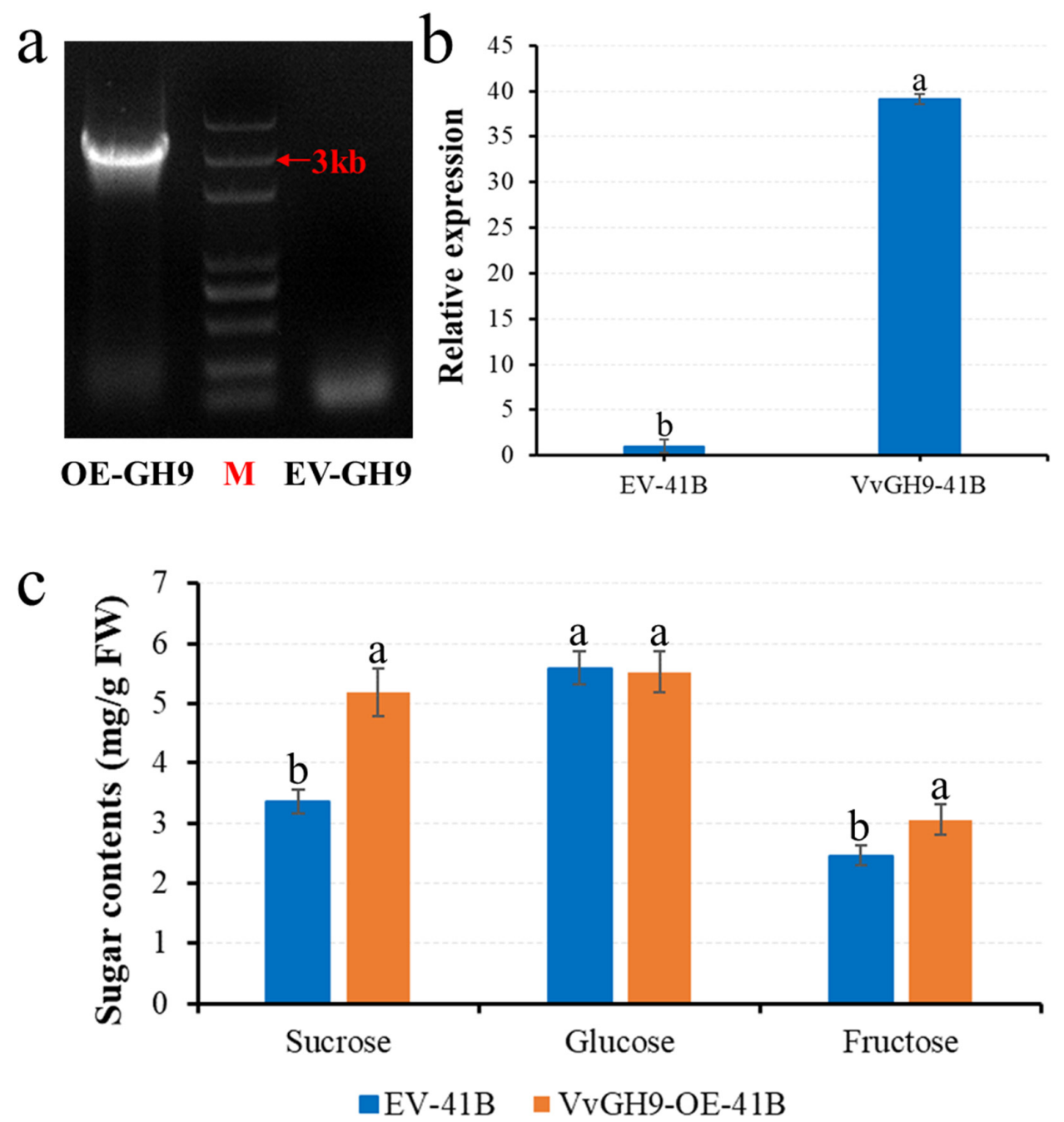

Figure 8. $V v G H 9$ overexpression and phenotypic identification of grape calli. (a) Verification of $V v G H 9$ insertion in the transgenic 41B calli. (b) The upregulation of GH9-OE in 41B calli was determined by qRT-PCR. (c) Analysis of sugar levels in EV and GH9-OE calli. FW: fresh weight. The letters above the bars indicated the significant differences by student's $t$-test $(p<0.05)$. Three biological replicates were analyzed, and the error bars represented the SD.
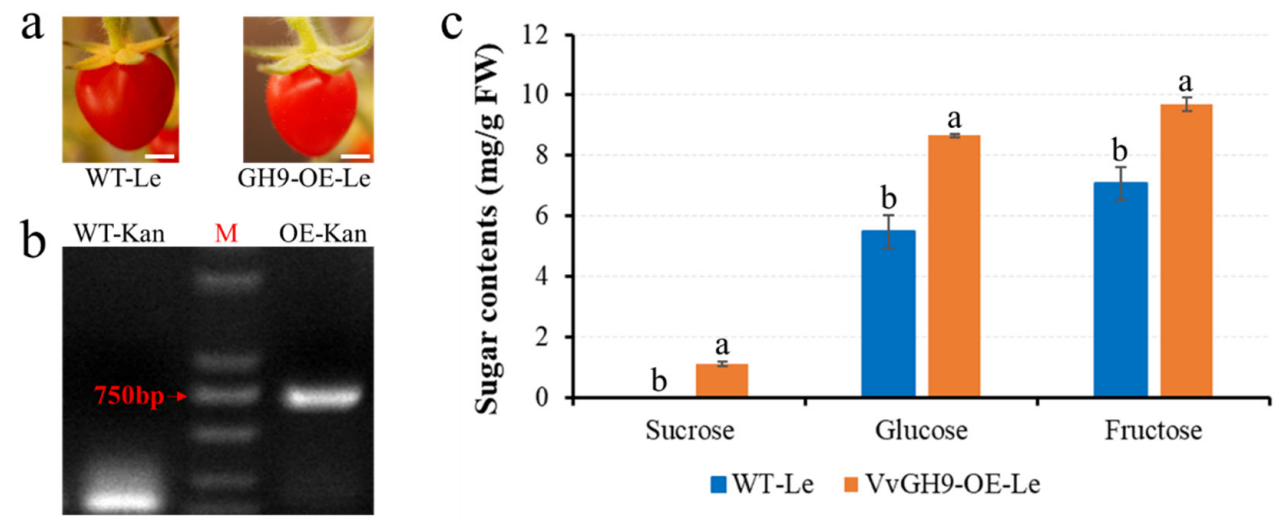

Figure 9. VvGH9 overexpression and phenotypic identification of tomato (Lycopersicon esculentum) fruits. (a) The left panel shows the ripe fruit of the wild-type tomato (WT-Le), and the right panel is the ripe fruit of overexpressing VvGH9 (VvGH9OE-Le). Scale bars, $1 \mathrm{~cm}$. (b) Verification of $V v G H 9$ insertion in the transgenic tomatoes. Kan: kanamycin. (c) Analysis of sugar levels in WT-Le and VvGH9-OE-Le. FW: fresh weight. The letters above the bars indicated the significant differences by student's $t$-test $(p<0.05)$. Three biological replicates were analyzed, and the error bars represented the SD. 


\section{Discussion}

In the present study, we identified $11 \mathrm{GH}$ genes divided into four groups in the grapevine genome, and examined the expression patterns across the different developmental stages of 22 grape varieties. Finally, VvGH9 was found to contribute to increased sugar content in plant and its function was verified by the transgene overexpression approach. The obtained results provide the foundation for future functional analysis of individual $V v G H$ gene.

Gene duplication events are important reasons for the expansion of the gene family, which include segmental and tandem duplication [32]. According to the whole genome duplication and tandem repeat analysis, eight $V v G H s$ were involved in three segmental duplication events and four $V v G H s$ were involved in two tandem duplication events, which implied that the segment duplication and tandem duplication all contribute to the $V v G H$ family expansion.

Recent studies of GH proteins mainly focused on their enzyme structures and involvement in sugar metabolism. The first enzyme structure of a glycosyl hydrolase, which was a lysozyme of hen egg white, was cracked more than 56 years ago [33]. Henrissat et al. [34] proposed that more than 45 families of glycosyl hydrolases were classified by their similarities of amino acid sequence. Nomenclature for sugar-binding subsites of glycosyl hydrolases was first proposed by Davies et al. [35]. White et al. [36] elucidated the mechanism of polysaccharide hydrolysis by retaining $\beta$-glycosyl hydrolases. In one recent report, the loss-of-function mutants of GH43 in Arabidopsis thaliana exhibited expansion defection for root cells [37]. In addition, alternative splicing event formed a shorter isoform of $\beta$-D-glucosidase, a GH1 family member, leading to a completely lack its activity in Catharanthus roseus [38]. However, there was almost no report that $G H$ genes involved in sugar accumulation in plants.

Since gene expression patterns can provide important clues for gene function, we then investigated the expression level of $V v G H s$ in different developmental stages of 22 grape varieties [27]. The results revealed that most $V v G H$ genes mainly played vital roles at the early stages of grape berry development. More so, the expression level of $V v G H 9$ was detected to be markedly different at three developmental stages among almost all the 22 grape varieties, and its expression was higher in early development stages (FS) with low sugar contents of the grape berry. In addition, $V v G H 5, V v G H 6$, and $V v G H 9$ had similar expression patterns, while only $V v G H 9$ was expressed and existed marked differential expression at three stages of all the six selected cultivars. Meanwhile, five different developmental stages of 'Muscat Hamburg' berries were chosen to measure sugar contents and to further verify $V v G H 9$ expression patterns via a qRT-PCR assay, which also implied that $\mathrm{VvGH9}$ might be involved in the sugar metabolism and accumulation of grape berries. Further experiments on grape fruits treated with exogenous sugar showed that $V v G H 9$ responded to the supplemental sucrose, glucose, and fructose, and responded most quickly and strongly to sucrose, which suggested that it may be involved in the hydrolysis of sucrose into monosaccharides. On the other hand, treatment of ' $41 \mathrm{~B}^{\prime}$ ' with different glucose concentrations indicated that the expression level of $\mathrm{VvGH9}$ was promoted by the increasing sugar concentrations lower than $30 \mathrm{mg} / \mathrm{g}$ and its expression was inhibited by higher sugar concentrations. Combined with the analysis of $V v G H 9$ expressions and sugar content evidence, we concluded that $V v G H 9$ may play an important role in promoting sugar metabolism and accumulation of fruits under low sugar content.

Overexpression of $\mathrm{VvGH} 9$ both in grape calli and tomato fruit resulted in a marked increase of sucrose, fructose, and total sugar (all the three soluble sugars detected in this study) content. In addition, sucrose was produced in VvGH9-OE-Le, while the wild-type tomato contained almost no sucrose, suggesting that $V v G H$ may play a vital role in the hydrolysis of polysaccharide to disaccharide. However, the contents of all three sugars were less than $10 \mathrm{mg} / \mathrm{g}$ in all the transgenic materials, which means that $V v G H 9$ may work in low sugar content. 
Based on all of these results above, we draw a preliminary conclusion that VvGH9 in grapevine might participate in sugar metabolism and accumulation under low sugar content. The different expression patterns of the different $G H$ genes indicated that each might play a specific and unique role. This study may provide some theoretical basis and experimental methods for exploring the physiological functions and molecular mechanisms of other $G H$ genes.

\section{Conclusions}

The GH gene family of the grape contains 11 members divided into four groups and is unevenly distributed on seven chromosomes. Most $V v G H$ members exhibited significant expression difference between different stages, while only $V v G H 9$ had observable differences in the three stages of almost all the 22 grape varieties. The expression patterns of $\mathrm{VvGH9}$ in different stages of grape fruit and in the materials treated with exogenous sugar revealed that $V v G H 9$ was induced by the increase of sugar with low content and was inhibited by high sugar content. Overexpressed $V v G H 9$ in grape calli and tomatoes further proved that it could promote sugar accumulation under low sugar content. This study is a new perspective on an important grape gene family for the molecular breeding of grape sugar in the future.

Supplementary Materials: The following are available online at https:/ / www.mdpi.com/article/ 10.3390/horticulturae7110453/s1, Table S1: Names and backgrounds of different grape varieties corresponding to different acronyms, Table S2: RNA-Seq data of the $11 \mathrm{VvGH}$ members in 22 grape cultivars, Table S3: List of primers used in this study.

Author Contributions: Conceptualization, Z.L.; methodology, G.X.; software, Y.W. (Yi Wang); validation, G.X., C.R. and P.F.; formal analysis, G.X. and Y.W. (Yi Wang); investigation, Y.K. and Y.W. (Yue Wang); resources, P.F. and Z.L.; data curation, G.X. and Y.W. (Yi Wang); writing-original draft preparation, G.X. and Y.W. (Yi Wang); writing-review and editing, G.X., Y.W. (Yi Wang), C.R. and Z.L.; visualization, G.X. and Y.W. (Yi Wang); supervision, P.F. and Z.L.; project administration, Z.L.; funding acquisition, Z.L. All authors have read and agreed to the published version of the manuscript.

Funding: This research was funded by the Strategic Priority Research Program of Chinese Academy of Sciences (Grant No. XDA24030404-3), the National Key Research and Development Program of China (Grant No. 2018YFD1000105), China-Africa Center for Research and Education, Chinese Academy of Sciences (Grant No. 151111KYSB20200017), and the Alliance of International Science Organizations (Grant No. ANSO-CR-PP-2020-04).

Institutional Review Board Statement: Not applicable.

Informed Consent Statement: Not applicable.

Data Availability Statement: All data in this study could be found in the manuscript or supplemental materials.

Conflicts of Interest: All the authors declare no conflict of interests.

\section{References}

1. Zhou, M.; Li, Y.F.; Liu, T.X.; Yang, G.S. Research progress in sugar accumulation in grape berries. Hunan Agric. Sci. 2020, 11, 91-95.

2. Zhang, Q.; Chen, Q.S.; Liu, Y.T.; Yin, P.; Meng, Z.F. Research progress on saccharide composition and quality characters of grape fruit. Hubei Agric. Sci. 2012, 51, 4978-4981.

3. Roitsch, T. Source-sink regulation by sugar and stress. Curr. Opin. Plant Biol. 1999, 2, 198-206. [CrossRef]

4. Henrissat, B.; Callebaut, I.; Fabrega, S.; Lehn, P.; Mornon, J.P.; Davies, G. Conserved catalytic machinery and the prediction of a common fold for several families of glycosyl hydrolases. Proc. Natl. Acad. Sci. USA 1995, 92, 7090-7094. [CrossRef] [PubMed]

5. McCarter, J.D.; Withers, S.G. Mechanisms of enzymatic glycoside hydrolysis. Curr. Opin. Struct. Biol. 1994, 4, 885-892. [CrossRef]

6. Semenza, G.; Auricchio, S. Small-intestinal disaccharidases. In The Metabolic Basis of Inherited Diseases; Scrives, C.R., Beaudet, A.L., Eds.; McGraw-Hill: New York, NY, USA, 1989; pp. 2975-2977.

7. Neufeld, E.F. Lysosomal storage diseases. Annu. Rev. Biochem. 1991, 60, 257-280. [CrossRef]

8. Bauer, M.W.; Driskill, L.E.; Kelly, R.M. Glycosyl hydrolases from hyperthermophilic microorganisms. Curr. Opin. Biotechnol. 1998, 9, 141-145. [CrossRef] 
9. Naumoff, D.G. Conserved sequence motifs in levansucrases and bifunctional $\beta$-xylosidases and $\alpha$-L-arabinases. FEBS Lett. 1999, 448, 177-179. [CrossRef]

10. Ermakova, S.P.; Ivanova, E.P.; Bakunina, I.Y.; Mikhailov, V.V.; Zvyagintseva, T.N. Effect of Brown Algae metabolites on the synthesis of O-glycosyl hydrolases by bacteria degrading the thallus of Fucus evanescens. Microbiology 2012, 81, 396-402. [CrossRef]

11. Sathya, T.A.; Khan, M. Diversity of glycosyl hydrolase enzymes from metagenome and their application in food industry. J. Food Sci. 2014, 79, 11. [CrossRef]

12. Chen, H.C.; Jin, X.; Zhu, L.J.; Lu, Y.L.; Ma, Z.; Liu, S.J.; Chen, X.L. Glycosyl hydrolase catalyzed glycosylation in unconventional media. Appl. Microbiol. Biotechnol. 2020, 104, 9523-9534. [CrossRef]

13. Bian, Z.; Wang, D.L.; Liu, Y.S.; Xi, Y.M.; Wang, X.L.; Meng, S. Analysis of Populus glycosyl hydrolase family I members and their potential role in the ABA treatment and drought stress response. Plant Physiol. Biochem. 2021, 163, 178-188. [CrossRef]

14. Yang, J.F.; Ma, L.; Jiang, W.B.; Yao, Y.; Tang, Y.H.; Pang, Y.Z. Comprehensive identification and characterization of abiotic stress and hormone responsive glycosyl hydrolase family 1 genes in Medicago truncatula. Plant Physiol. Biochem. 2021, 158, 21-33. [CrossRef] [PubMed]

15. Bauer, S.; Vasu, P.; Persson, S.; Mort, A.J.; Somerville, C.R. Development and application of a suite of polysaccharide-degrading enzymes for analyzing plant cell walls. Proc. Natl. Acad. Sci. USA 2006, 103, 11417-11422. [CrossRef]

16. Bourne, Y.; Henrissat, B. Glycoside hydrolases and glycosyltransferases: Families and functional modules. Curr. Opin. Struct. Biol. 2001, 11, 593-600. [CrossRef]

17. Tricone, A. Uncommon glycosidases for the enzymatic preparation of glycosides. Biomolecules 2015, 5, 2160-2183. [CrossRef] [PubMed]

18. Stevenson, D.G.; Domoto, P.A.; Jane, J.L. Structures and functional properties of apple (Malus. domestica Borkh) fruit starch. Carbohydr. Polym. 2006, 63, 432-441. [CrossRef]

19. Bertin, N.; Causse, M.; Brunel, B.; Tricon, D.; Génard, M. Identification of growth processes involved in QTLs for tomato fruit size and composition. J. Exp. Bot. 2009, 60, 237-248. [CrossRef] [PubMed]

20. Hu, X.; Kuang, S.; Zhang, A.D.; Zhang, W.S.; Chen, M.J.; Yin, X.R.; Chen, K.S. Characterization of starch degradation related genes in postharvest Kiwifruit. Int. J. Mol. Sci. 2016, 17, 2112. [CrossRef]

21. Sinha, S.K.; Datta, S. $\beta$-Glucosidase from the hyperthermophilic archaeon Thermococcus sp. Is a salt-tolerant enzyme that is stabilized by its reaction product glucose. Appl. Microbiol. Biotechnol. 2016, 100, 8399-8409. [CrossRef] [PubMed]

22. Jiang, Z.D.; Long, L.F.; Liang, M.F.; Li, H.B.; Chen, Y.H.; Zheng, M.J.; Ni, H.; Li, Q.B.; Zhu, Y.B. Characterization of a glucosestimulated $\beta$-glucosidases from Microbulbifer sp. ALW1. Microbiol. Res. 2021, 251, 126840. [CrossRef] [PubMed]

23. Wang, Z.M.; Wang, Y.; Tong, Q.; Xu, G.Z.; Xu, M.L.; Li, S.H.; Fan, P.G.; Li, P.G.; Liang, Z.C. Transcriptomic analysis of grapevine Dof transcription factor gene family in response to cold stress and functional analyses of the VaDof17d gene. Planta 2021, 253, 55. [CrossRef] [PubMed]

24. Di, F.F.; Jian, H.J.; Wang, T.Y.; Chen, X.P.; Ding, Y.R.; Du, H.; Lu, K.; Li, J.N.; Liu, L.Z. Genome-wide analysis of the PYL gene family and identification of PYL genes that respond to abiotic stress in Brassica napus. Genes 2018, 9, 156. [CrossRef]

25. Xu, M.L.; Tong, Q.; Wang, Y.; Wang, Z.M.; Xu, G.Z.; Elias, G.K.; Li, S.H.; Liang, Z.C. Transcriptomic Analysis of the Grapevine LEA Gene Family in Response to Osmotic and Cold Stress Reveals a Key Role for VamDHN3. Plant Cell Physiol. 2020, 61, 775-786. [CrossRef]

26. Krzywinski, M.; Schein, J.; Birol, I.; Connors, J.; Gascoyne, R.; Horsman, D.; Jones, S.J.; Marra, M.A. Circos: An information aesthetic for comparative genomics. Genome Res. 2009, 19, 1639-1645. [CrossRef]

27. Wang, Y.; Zhang, R.; Liang, Z.C.; Li, S.H. Grape-RNA: A database for the collection, evaluation, treatment, and data sharing of grape RNA-seq datasets. Genes 2020, 11, 315. [CrossRef] [PubMed]

28. Jaillon, O.; Aury, J.M.; Noel, B.; Policriti, A.; Clepet, C.; Casagrande, A.; Choisne, N.; Aubourg, S.; Vitulo, N.; Jubin, C.; et al. The grapevine genome sequence suggests ancestral hexaploidization in major angiosperm phyla. Nature 2007, 449, 463-467.

29. Sun, H.J.; Uchii, S.; Watanabe, S.; Ezura, H. A highly efficient transformation protocol for Micro-Tom, a model cultivar for tomato functional genomics. Plant Cell Physiol. 2006, 47, 426-431. [CrossRef]

30. Zhang, Z.; Zou, L.M.; Ren, C.; Ren, F.R.; Wang, Y.; Fan, P.G.; Li, S.H.; Liang, Z.C. VvSWEET10 mediates sugar accumulation in grapes. Genes 2019, 10, 255. [CrossRef]

31. Coombe, B.G. Growth Stages of the Grapevine: Adoption of a system for identifying grapevine growth stages. Aust. J. Grape Wine Res. 1995, 1, 104-110. [CrossRef]

32. Cannon, S.B.; Mitra, A.; Baumgarten, A.; Young, N.D.; May, G. The roles of segmental and tandem gene duplication in the evolution of large gene families in Arabidopsis thaliana. BMC Plant Biol. 2004, 4, 10. [CrossRef]

33. Blake, C.C.F.; Koening, D.F.; Mair, G.A.; North, A.C.T.; Phillips, D.C.; Sarma, V.R. Structure of hen egg white lysozyme. A three-dimensional Fourier synthesis at 2 Å resolution. Nature 1965, 206, 757-763. [CrossRef] [PubMed]

34. Henrissat, B.; Bairoch, A. New families in the classification of glycosyl hydrolases based on amino acid sequence similarities. Biochem. J. 1993, 293, 781-788. [CrossRef] [PubMed]

35. Davies, G.J.; Wilson, K.S.; Henrissat, B. Nomenclature for sugar-binding subsites in glycosyl hydrolases. Biochem. J. 1997, 321, 557-559. [CrossRef]

36. White, A.; Rose, D.R. Mechanism of catalysis by retaining $\beta$-glycosyl hydrolases. Curr. Opin. Struct. Biol. 1997, 7, 645-651. [CrossRef] 
37. Nibbering, P.; Petersen, B.; Motawia, M.S.; Jorgensen, B.; Ulvskov, P.; Niittyla, T. Golgi-localized exo- $\beta 1$,3-galactosidases involved in cell expansion and root growth in Arabidopsis. J. Biol. Chem. 2020, 295, 10581-10592. [CrossRef] [PubMed]

38. Carqueijeiro, I.; Koudounas, K.; Bernonvile, T.D.; Sepulveda, L.J.; Mosquera, A.; Bomzan, D.P.; Oudin, A.; Lanoue, A.; Besseau, S.; Cruz, P.L.; et al. Alternative splicing creates a pseudo-strictosidine $\beta$-D-glucosidase modulating alkaloid synthesis in Catharanthus roseus. Plant Physiol. 2021, 185, 836-856. [CrossRef] [PubMed] 